\title{
Information Security Technology under Cloud Computing Technology
}

\author{
Zhao Xiaojin \\ Hainan Vocational College Of Political Science And Law
}

\begin{abstract}
Keywords: Cloud computing; Information security;
\end{abstract}
\begin{abstract}
With the coming of information era, cloud computing has become a strong pillar for important management, storage, etc. in modern society development. In people's development course, its application has also been in unprecedented development speed. In the process of this development, through collecting summary of global information, it further completed the response construction of modern economic globalization so as to realize the definition of the overall information through comprehensive information construction, which completes the final guidance of cloud computing information. In the application development of information computing, it can also further perfect the comprehensive construction of cloud computing application information security and promote global security implementation. Based on information security applications, this paper gives a brief analysis on information security technology application in cloud computing technology.

With the continuous development of modern science and technology, the concept of cloud computing was put forward and in the field of information technology, it has become the key to realize in fields of modern industry, life, etc. But the security of cloud computing technology has also become one of the system's most controversial points. Because of safety problems influence, the research object in the field of computer science is in dispute. To complete the application of operation maintenance service management, on the concept, strengthen the focus from all walks of life on cloud computing technology, and according to the actual production application, make effective information regulation. According to production application, make effective information adjustment. According to production security challenges form, complete the comprehensive information construction of cloud computing. Here this paper briefly argues from the information security technology perspective of cloud computing technology.
\end{abstract}

\section{THE INTRODUCTION OF CLOUD COMPUTING TECHNOLOGY}

Cloud computing technology is a terminal mode construction in the process of the development of science and technology, which achieves access construction to information resources through network structure model, and further completes the redistribution of management cost through the regulation of network and service resources. So with the original economic development, at the same time, it further promotes the individual development of economic units and becomes one of important compositions for modern social development.

Cloud computing system framework has five main features, respectively, elastic, and on-demand self-service, billing services, independent resources and information access. In the process of management implementation, the information application construction needs to conduct a comprehensive analysis, and through data adjustment, fundamentally achieves operation scheduling of the overall information.

\section{THE SECURITY SITUATION FOR CLOUD COMPUTING SYSTEM}

Along with the development of modern science and technology, in social management with cloud computing application, network share storage calculation has many problems in many fields, and its security has to be promoted. From all internal information adjustment of computing system and traditional system, complete the effective management of safety problems through data loss and service application. The security issues in the society development are mainly the followings. 


\section{A. Trust boundary problem}

With the marketization of IT pattern, IT resources have become the main monitoring center of modern social units. Implement security isolation access with this border system, and on the basis of the theory's security applications, complete the center level integration of cloud computing data, and realize the system management with resources data application of store computing. But in the implementation process of this concept, the system has had great changes, which leads to the original boundary change and eventually leads to safety management form change. Thus it affects data security management framework.

B. Data security problem

In view of data security issues, cloud computing model changes lead to the change of application data concentration. Illegal personnel's illegal operation of the information could lead to adverse supply to internal information, such as hacking, etc., and for information data privacy of application system, the security risk is affected seriously.

\section{System usability problems}

As is known to all, cloud computing system model has its larger application data and information database is from scattered to centralized, which lead to some information data loss for some reason. The usability problems, therefore, may lose because of the core issues, which may seriously affect the safe operation of cloud computing system and the actual service function.

\section{THE SOLUTION EXPLORATION FOR CLOUD COMPUTING INFORMATION SECURITY PROBLEMS}

Accompanied by the emergence of many problems, the advantages of cloud computing system is still to be reckoned with. As long as these defects are ruled out, they can better promote the development of modern society. In the process of solving these problems, much information security correction, such as data integration encryption processing and identity authentication and so on, will be able to really make sure to its information security. From the implementation of specific measures, extend implementation monitoring of data flow and operation status of cloud computing platform. After discovering network anomalies, pilot timely processing. Virtual machines VM image protection shall also be authorized to access measures, and strengthen the damaging effects of information according to actual virus modify policies to prevent foreign information virus to infect a computer system. Under the influence of the concept, strengthen all code supply security through strengthening the deployment of computer defense system, establish good identity authentication, and strictly enforce watchdog for identity management through security information log in to provide users with more access possibility and complete the backup operation of calculation information. Accommodate the backup of system information data need to be protected, and establish a more perfect corresponding mechanism. Through emergency corresponding protection, realize the effective protection of the overall information.

\section{CLOUD COMPUTING INFORMATION SECURITY DESIGN ANALYSIS}

In modern traditional operation of cloud computing environment, strengthen the protection of border protection. Based on the safety characteristics of cloud computing environment, introduce safety protection which can meet the demand of production technology and to safeguard the security of cloud computing platform. For the virtual security form, strengthen the security authentication of user information from host system security data and realize the effective protection for network attack, information transmission and other aspects through network transmission security management. For this, in the process of protection implementation, brief analysis is done through the following strategies designs.

A. Physical security

On the design of physical information security, carry out effective construction of computer center statistics according to the security of data center, and make high safety whole computer room construction according to the constructed availability information to ensure the security of system hardware software and prevent the invasion of foreign information interference. In view of 
environmental factors' information consumption, strengthen the security construction of physical safety information, and realize the hardware equipment troubleshooting through the configuration management of equipment malfunction and do a comprehensive review of fault management in time. To run the configuration fault detection work, we need to avoid single fault service work suspension, and construct an effective protection according to the actual fault condition, and according to the machine room management system, strengthen the application of service management. To power supply disruption risk problem, multi-channel power supply system should also be applied to provide uninterrupted power system to supply power system stability. For safety management measures of physical system, establish a natural environment resistance to protect comprehensive security backup, and avoid the continuity damage of computing services caused by earthquake, fire, water and other accidents.

B. Virtual information security

Application of cloud computing technology mainly aims at virtual information service. Complete the application of electronic circuits' implementation through virtual information service, and eventually achieve the effective implementation of the real work. The information application also promotes the effective application of information operation. Therefore a good job in security applications of virtual information management basically realizes the security applications of virtualization information, and through safe operation environmental protection inside virtual machine, basically realizes mechanics internal security information backup. Realize effective guarantee for information's overall organization through information protection so as to realize the effective protection of virtual machine's internal structure.

\section{Host system security}

In the process of cloud computing system application, you need to make sure the client security within the host system, and completes the safety implementation of the host platform. Do a good client's safety job and prevent problems caused by virus system virtualization patch structure. Prevent the internal information security applications of host platform. The decision information should be consistent with the actual operation requirements and take users' input and output information mode. Effectively complete the comprehensive application of information detection range.

\section{User data storage}

In order to ensure the security and integrity of data, it is necessary to strengthen the data security storage permissions settings and ensure the independence of user information through the isolation application of the stored information. Finally realize the security protection function. The extraction reading of information also needs to clean up the information through data access and make inspection operation.

\section{E. User information access operation security and authentication}

User information access and remote operation should be after the confirmation of user information and through identity information's unified employment, realize effective access to user account information, and strengthen the implementation of information application. Through effective information control, effectively complete user privilege information access application and further implement the planning application of user access through account control.

F. Network security management measures

Cloud computing service objects are mainly from network information, so the safety management of network information can guarantee the safety of information service. The security application problems of network information are mainly focused on good optimization measures of information system, information structure adjustment, the planning application strengthening of network link channel and network structure, and attack protection from malicious codes to complete of Internet broadband information prevention management. When facing network virtual attack, strengthen the information cutting processing of the virtual machine to prevent remote vulnerabilities, and work to strengthen the protection of denial service. The storage medium's virus transmission problem should be handed over to the relevant departments to conduct a comprehensive information investigation to prevent information problem caused by storage medium 
virus transmission.

G. Code development and safety management

Before the implementation of cloud computing services, the security system of original code should be set up independently to avoid the internal function application across problems, which lead to audit problems. Set legislation for engineering audit process which also needs to strengthen the agreement application of the cloud computing platforms, and include it in the enterprise's control management system.

H. Establish a sound legal act

In the implementing process of system safety management, strengthen effective information creation. Set up a sound information security protection system by judicial organizations, apply laws to protect the interests of operators and customers and put an end to the illegal invasion problem.

\section{CONCLUSION}

With the rapid development of modern society, in the process of new type computer application implementation, improve the enterprise's information application system construction through Internet application services. Provide a new social system more in form of social development for the future effective information construction development. With cloud computing system as the carrier, traditional information service system will be completely reversed and the adjustment of security model also can apply the original development model to improve the overall plan construction of better facilities construction. Through effective information creation, further improve information security construction.

\section{REFERENCES}

[1] Hui Zhibin. Cloud computing information security theory and countermeasure research in China [J]. Science and Technology Management Research, 2013, (16) : 171-174180.

[2] Pan Xiaoming, Zhang Xiangyang, Shen Xiyong. Cloud computing information security evaluation framework research [J]. Journal of Computer Age, 2013, (10) : 22-25.

[3] Yang Xuejun. Framework design of cloud computing information security evaluation [J]. Science and Technology of China Horizon, 2014, (14) : 41 and 42.

[4] Wang Shiwei, zhao Fuchun. Cloud computing information security policy research under enterprise users perception perspective [J]. Journal of Library Intelligence, 2012, 56 (6) : 29-32.

[5] Du Baodong, Yang Qingming, Li Bing. Enterprise cloud computing information safety plan research [J]. Information Systems Engineering, 2014, (5) : 67-68.

[6] Zhang Wei. Cloud computing power enterprise information security risk assessment study [J]. Guangdong Science and Technology, 2013, (20) : 49 and 50,75.

[7] Hou Hongfeng, Wang Can, Wang Lijuan. Cloud computing information security problems study [J]. Journal of Electronic Design Engineering, 2012, 20 (22) : 60-62. 